\title{
ADSORPTION WITH CHEMICAL REACTION IN POROUS CATALYST PELLETS UNDER ALTERNATE CONCENTRATION FIELDS. UNIFORM TEMPERATURE CASE
}

\author{
Katarzyna Bizon, Bolesław Tabiś* \\ Cracow University of Technology, Department of Chemical and Process Engineering, \\ ul. Warszawska 24, 31-155 Kraków, Poland
}

\begin{abstract}
The work concerns the dynamic behaviour of a porous, isothermal catalyst pellet in which a simultaneous chemical reaction, diffusion and adsorption take place. The impact of the reactant adsorption onto the pellet dynamics was evaluated. A linear isotherm and a non-linear Freundlich isotherm were considered. Responses of the pellet to sinusoidal variations of the reactant concentration in a bulk gas were examined. It was demonstrated that the dynamics of the pellet is significantly affected both by accounting for the adsorption and by the frequency of the bulk concentration variations. The sorption phenomenon causes damping of the concentration oscillations inside the pellet and damping of its effectiveness factor oscillations. Depending on the frequency of the concentration oscillations in the bulk, the remarkable oscillations can involve an entire volume of the pellet or its portion in the vicinity of the external surface.
\end{abstract}

Keywords: catalyst pellets, reaction, diffusion, adsorption, dynamics

\section{INTRODUCTION}

Determination of a steady-state characteristics of a single catalyst pellet or the whole catalytic reactor does not require accounting for the mass accumulation within pellets due to the adsorption. However, at transient conditions the reaction rate can differ from the reactant supply rate. In such a case mass accumulation within the pellet occurs. Therefore, when external perturbations of the bulk reactant concentration take place, the mass balance must include both a term referring to the mass accumulation in pores and an adsorption term characterizing the mass accumulation on the internal surface of the inert catalyst support. Such an opinion was also expressed by Ray and Hastings (1980). Ilin and Luss (1992) analysed the dynamics of a fixed-bed catalytic reactor. The authors also believe that dynamic models should account for the reactant adsorption on the inert support of porous catalyst pellets. Ilin and Luss (1992) neglected internal resistances to mass and heat transfer in catalyst pellets. Nevertheless, despite simplicity of the model employed they demonstrated that taking into account the reactant sorption affects dynamics of the reactor.

Still, some authors do not consider the mass accumulation by adsorption in the characterization of the catalyst pellets dynamics. Examples of such a modelling are works of Szukiewicz (2002) or Burghardt and Berezowski (2003).

In hybrid, i.e. multifunctional, catalyst pellets the presence of the adsorbent is intentional and is resulting from the production technology and their application (Dietrich et al., 2005; Grünewald and 
Agar, 2004; Lugo and Wilhite, 2016). Therefore taking into account the sorption of reagents in the dynamics of such objects is fully justified. For uniformly distributed design the modelling approach of the multifunctional porous catalyst pellets is the same as for conventional catalyst pellets composed of an inert support and an active substance.

Considering the papers published so far, we undertook the studies focused on the impact of the sorption phenomenon of the reactant onto the dynamics of a single catalyst pellet. When the bulk reactant concentration increases, for example due to an external factor, then an external mass transfer and an internal mass diffusion within the pellet may increase the reactant concentration above the steady-state value. A dynamic variation of the reactant concentration in turn influences the rate of a chemical reaction. The time necessary to reach a new steady state depends on the quantity of the adsorbed reactant and on the rates of diffusion and reaction. The opposite case consisting in a decrease in the bulk concentration or in an oscillatory variation of the reactant concentration may also occur. All these operational conditions require an evaluation of the impact of mass accumulation within the pellet onto its dynamic characteristics.

The work concerns a catalytic process for which an assumption of the uniform pellet temperature is made. In fact many authors believe that the temperature distribution in the porous catalyst pellet is negligible when compared with the concentration distribution (Burghardt and Berezowski, 2003; Ilin and Luss, 1992; Lee, 1985). Burghardt and Berezowski (2003) assumed uniform distribution of the temperature within the pellet, although they considered its variation with time.

Variations of the bulk concentration can be divided into: step, periodical and irregular. In the present study we investigated the transient response of the pellet to regular, sinusoidal variations of the bulk reactant concentration. Both internal and external resistances to the mass transfer were considered. The type of the sorption equilibrium and the frequency of oscillations in the bulk were taken into account in the analysis. The main objective of the work was to answer the question: how the reactant concentration within the pellet and its effectiveness factor change upon periodic external perturbations. The results of such theoretical investigations have not been published so far.

\section{ADSORPTION AND CHEMICAL REACTION IN A POROUS CATALYST PELLET}

According to heterogeneous models of a catalyst bed, separate balance equations must be written for a gas phase and for catalyst pellets (Fig. 1).

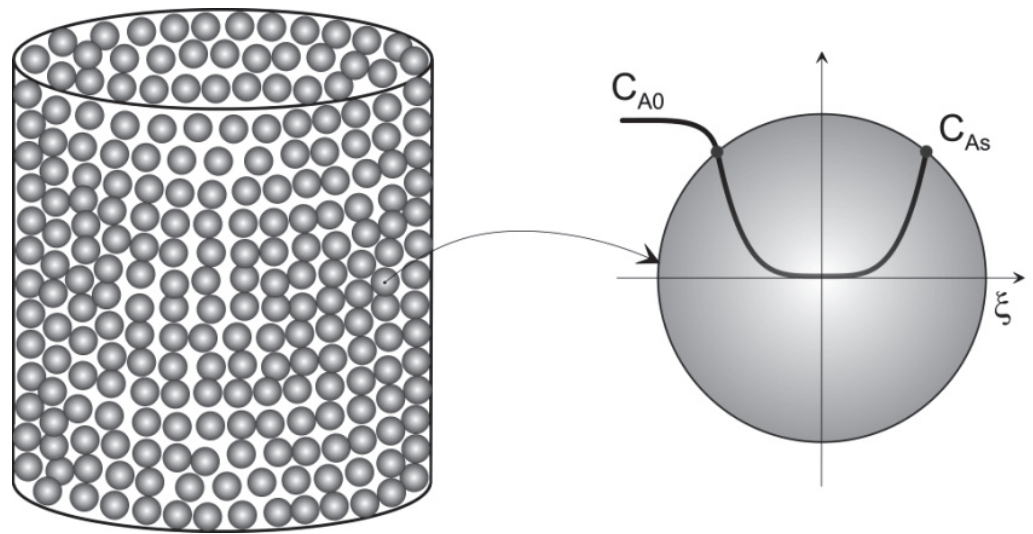

Fig. 1. Illustration of heterogeneous nature of the catalyst bed with basic notation

Let us consider a catalyst pellet characterized by a uniform temperature in which a chemical reaction described by the following kinetic rate expression takes place 


$$
r_{\mathrm{A}}(C)=k \cdot \varphi(C)
$$

where $C$ is a vector of the reactant concentrations present in the kinetic model. Let us assume that the chemical process may be accompanied by the reactant adsorption or desorption. There is an opinion (Szarawara and Skrzypek, 1980) that a surface diffusion does not play an important role in catalytic processes. Thus, under such a hypothesis, the mass balance equation of a component A over a differential volume element of the pellet can be written as follows

$$
\varepsilon_{p} \frac{\partial C_{\mathrm{A}}}{\partial t}+\rho_{p} \frac{\partial q}{\partial t}=D_{e}\left(\frac{\partial^{2} C_{\mathrm{A}}}{\partial x^{2}}+\frac{n}{x} \frac{\partial C_{\mathrm{A}}}{\partial x}\right)-r_{\mathrm{A}}(C)
$$

where $n=0,1$, or 2 are shape factors for slab, cylinder and sphere, respectively. If we write the derivative $\partial q / \partial t$ as

$$
\frac{\partial q}{\partial t}=\frac{d q}{d C_{\mathrm{A}}} \frac{\partial C_{\mathrm{A}}}{\partial t}
$$

and we introduce it into the differential equation (2), this equation can be rewritten as

$$
\frac{\partial C_{\mathrm{A}}}{\partial t}=\frac{1}{\varepsilon_{p}+\rho_{p} \frac{d q}{d C_{\mathrm{A}}}}\left[D_{e} \cdot\left(\frac{\partial^{2} C_{\mathrm{A}}}{\partial x^{2}}+\frac{n}{x} \frac{\partial C_{\mathrm{A}}}{\partial x}\right)-r_{\mathrm{A}}(C)\right]
$$

In Eq. (4) the derivative $\frac{d q}{d C_{\mathrm{A}}}=K_{a}$ corresponds to a slope of the sorption equilibrium curve.

An irreversible $m^{\text {th }}$ order chemical reaction was adopted in numerical tests to illustrate the impact of the adsorption phenomenon on the pellet dynamics. Thus, the kinetic rate equation in Eq. (4) takes a simple form

$$
r_{\mathrm{A}}(C)=r_{\mathrm{A}}\left(C_{\mathrm{A}}\right)=k \cdot C_{\mathrm{A}}^{m}
$$

The initial condition associated with Equation (4) is given by

$$
C_{\mathrm{A}}(x, 0)=v_{0}(x), x \in\left[0, L_{p}\right]
$$

A symmetry of the concentration field about the centre of symmetry is assumed, namely about the sphere centre for $n=2$, about the longitudinal axis of the pellet for $n=1$ and about the plane of symmetry for $n=0$. Taking into account an external mass transfer resistance, boundary conditions at the pellet centre and at its surface take a form of Neumann-Robin (N-R) boundary conditions

$$
\begin{gathered}
\frac{\partial C_{\mathrm{A}}(0, t)}{\partial x}=0, t \geq 0 \\
D_{e} \frac{\partial C_{\mathrm{A}}\left(L_{p}, t\right)}{\partial x}=k_{s}\left[C_{\mathrm{A} 0}(t)-C_{\mathrm{A}}\left(L_{p}, t\right)\right], t \geq 0
\end{gathered}
$$

If we define the following dimensionless concentration within the pellet $\beta$ and the dimensionless coordinate $\xi$

$$
\beta=\frac{C_{\mathrm{A}}}{C_{\mathrm{A} r e f}} \in[0,1], \quad \xi=\frac{x}{L_{p}} \in[0,1]
$$

Eq. (4) with conditions (6) and (7) can be rewritten as 


$$
\begin{gathered}
\frac{\partial \beta}{\partial t}=\Gamma_{m} \cdot\left[\left(\frac{\partial^{2} \beta}{\partial \xi^{2}}+\frac{n}{\xi} \frac{\partial \beta}{\partial \xi}\right)-\Phi^{2} \frac{\widetilde{r}_{\mathrm{A}}(\beta)}{\widetilde{r}_{\mathrm{A} r e f}}\right] \\
\beta(\xi, 0)=\beta_{0}(\xi), \quad \xi \in[0,1] \\
\frac{\partial \beta(0, t)}{\partial \xi}=0, t \geq 0 \\
\frac{\partial \beta(1, t)}{\partial \xi}=\mathrm{Bi}_{m}[g(t)-\beta(1, t)], t \geq 0
\end{gathered}
$$

where $g(t)$ is a function describing variations of the bulk dimensionless reactant concentration, whereas $\widetilde{r}_{\mathrm{Aref}}=\widetilde{r}_{\mathrm{A}}\left(\beta_{r e f}\right)$ is a modified rate of a chemical reaction, i.e. $\widetilde{r}_{\mathrm{A}}(\beta)=\frac{r_{\mathrm{A}}\left(C_{\mathrm{A}}\right)}{C_{\mathrm{Aref}}}$, evaluated at the reference concentration $C_{\mathrm{A} \text { ref }}$. Since $C_{\mathrm{Aref}}$ corresponds to $\beta_{\text {ref }}=1$, thus for the chemical reaction of the $m^{\text {th }}$ order, used in our study we get $\widetilde{r}_{\mathrm{A} r e f}=C_{\mathrm{Aref}}^{m-1} \cdot k$.

In Eq. (9) the mass capacitance term, $\Gamma_{m}$, and the Thiele modulus, $\Phi$, are defined by

$$
\Gamma_{m}=\frac{D_{e}}{L_{p}^{2}} \frac{1}{\varepsilon_{p}+\rho_{p} K_{\mathrm{a}}}, \quad \Phi^{2}=L_{p}^{2} \frac{r_{\mathrm{A}}\left(C_{\mathrm{A} r e f}\right)}{C_{\mathrm{A} r e f} D_{e}}=L_{p}^{2} \frac{\widetilde{r}_{\mathrm{A} r e f}}{D_{e}}
$$

whereas the Biot number, $\mathrm{Bi}$, is defined as follows

$$
\mathrm{Bi}_{m}=\frac{L_{p} k_{s}}{D_{e}}
$$

It arises from Eq. (9) that the mass capacitance term has an impact only on the pellet dynamics. It does not affect the location of the steady-state branches. However, as demonstrated by Luss (1974), in case of a non-isothermal catalyst pellet for which the capacitance term is represented by the Lewis number, it can influence the stability of steady states.

\section{RESEARCH PROGRAMME}

Let us evaluate how the shape of the sorption isotherm influences the capacitance term. It results from the definition (12) that

$$
\Gamma_{m}=\frac{D_{e}}{L_{p}^{2}} \cdot \frac{1}{\varepsilon_{p}+\left(1-\varepsilon_{p}\right) \rho_{0} K_{a}}
$$

Two types of sorption equilibria were considered in the work, namely a linear isotherm and a nonlinear Freundlich isotherm (Fig. 2a, b).

In case of a linear isotherm characterized by specific values of the parameters for which

$$
\rho_{0} K_{a} \approx 1
$$

the capacitance term $\Gamma_{m}$ can be reduced to

$$
\Gamma_{m}=\frac{D_{e}}{L_{p}^{2}}
$$


When the exponent $v$ in the Freundlich isotherm equation tends to zero, then its shape approaches a rectangular (irreversible) isotherm. In such a case we obtain

$$
\lim _{v \rightarrow 0} \Gamma_{m}=\frac{D_{e}}{L_{p}^{2}} \frac{1}{\varepsilon_{p}}
$$
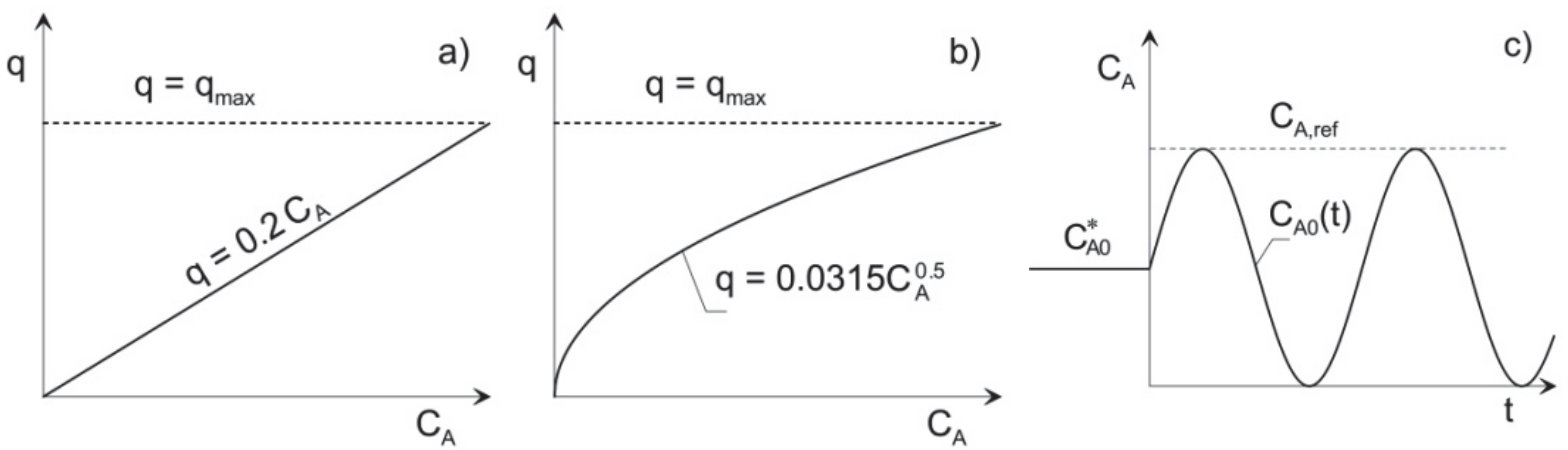

Fig. 2. Types of adsorption equilibria and external disturbance considered a) linear isotherm; b) Freundlich isotherm; c) external disturbance and used symbols

Parameter values used in numerical simulations are shown in Table 1. Only representative results are presented in the graphical form. The parameter values that refer to physico-chemical properties of the catalysts were selected based on the analysis of various technological processes, such as oxidation of $\mathrm{SO}_{2}$ to $\mathrm{SO}_{3}$, oxidation of selected organic compounds, methanol synthesis, hydrogenation of benzene to styrene or dehydrogenation of $\mathrm{C}_{2} \mathrm{H}_{5} \mathrm{OH}$ to $\left(\mathrm{C}_{2} \mathrm{H}_{5}\right)_{2} \mathrm{O}$.

Table 1. Values of the parameters employed in the numerical analysis of the pellet dynamics

\begin{tabular}{|c|c|c|c|}
\hline No & Parameter & Value & Dimension \\
\hline 1 & $k$ & 0.25 & $\mathrm{~m}^{3(m-1)} \cdot \mathrm{s}^{-1} \cdot \mathrm{kmol}^{(1-m)}$ \\
\hline 2 & $n$ & 2 & - \\
\hline 3 & $D_{e}$ & $10^{-6}$ & $\mathrm{~m}^{2} \cdot \mathrm{s}^{-1}$ \\
\hline 4 & $m$ & 1 & - \\
\hline 5 & $d_{p}$ & $8 \times 10^{-3}$ & $\mathrm{~m}$ \\
\hline 6 & $K_{a}$ & 0.2 & $\mathrm{~m}^{3} \cdot \mathrm{kg}^{-1}$ \\
\hline 7 & $K_{F}$ & 0.0315 & $\mathrm{~m}^{3 \nu} \cdot \mathrm{kg}^{-1} \cdot \mathrm{kmol}^{1-v}$ \\
\hline 8 & $v$ & 0.5 & - \\
\hline 9 & $q_{\max }$ & $5 \times 10^{-3}$ & $\mathrm{kmol} \cdot \mathrm{kg}^{-1}$ \\
\hline 10 & $\mathrm{Bi}_{m}$ & 5 & - \\
\hline 11 & $\rho_{p}$ & 1200 & $\mathrm{~kg} \cdot \mathrm{m}^{-3}$ \\
\hline 12 & $\omega$ & $0.001-0.1$ & $\mathrm{rad} \cdot \mathrm{s}^{-1}$ \\
\hline
\end{tabular}

The external disturbance consists of:

- determination of a steady state of the pellet,

- prescription of a function $g(t)$ and evaluation of the system response to this disturbance.

A sinusoidal variation of the bulk reactant concentration is given by the formula (18) (Fig. 1c)

$$
\frac{C_{\mathrm{A} 0}(t)}{C_{\mathrm{Aref}}}=g(t)=0.5 \cdot[1+\sin (\omega \cdot t)]
$$


The differential Eq. (9) with the conditions (10) and (11) was solved by means of a method of lines (Shampine, 1994). Spatial partial derivatives with respect to a variable $\xi$ were approximated using central finite difference schemes. Thus, the differential Eq. (9) with the boundary conditions (11) was transformed into a system of $N$ ordinary differential equations at $i=1, \ldots, N$ grid nodes along the interval $\xi \in[0,1]$.

For $i=1$, that is in the pellet centre $(\xi=0)$, considering the symmetry of the concentration profile (11a) we obtain

$$
\frac{d \beta_{1}}{d t}=\Gamma_{m} \cdot\left[\frac{2\left(\beta_{2}-\beta_{1}\right)}{\Delta \xi^{2}}-\Phi^{2} \frac{\widetilde{r}_{\mathrm{A}}\left(\beta_{1}\right)}{\widetilde{r}_{\mathrm{A} r e f}}\right]
$$

For subsequent nodes along the pellet radius we get

$$
\frac{d \beta_{i}}{d t}=\Gamma_{m} \cdot\left[\frac{\beta_{i+1}-2 \beta_{i}+\beta_{i-1}}{\Delta \xi^{2}}+\frac{1}{\xi} \frac{\beta_{i+1}-\beta_{i-1}}{\Delta \xi}-\Phi^{2} \frac{\widetilde{r}_{\mathrm{A}}\left(\beta_{i}\right)}{\widetilde{r}_{\text {Aref }}}\right], \quad(i=2, \ldots, N-1)
$$

At the pellet surface, i.e. at $\xi=1$, the boundary condition (11b) may be transformed in the following equation

$$
\frac{d \beta_{N}}{d t}=\Gamma_{m} \cdot\left[\frac{2\left[\beta_{N-1}+\Delta \xi \cdot \mathrm{Bi}_{m}\left(g(t)-\beta_{N}\right)-\beta_{N}\right]}{\Delta \xi^{2}}+2 \mathrm{Bi}_{m}\left(g(t)-\beta_{N}\right)-\Phi^{2} \frac{\widetilde{r}_{\mathrm{A}}\left(\beta_{N}\right)}{\widetilde{r}_{\mathrm{Aref}}}\right]
$$

The system of $N$ differential Eqs. (19) was integrated employing the method of Gear (1971) with the initial conditions

$$
\beta_{i}(0)=\beta_{0 i}, \quad(i=1, \ldots, N)
$$

In order to determine a response of the pellet to the bulk reactant concentration, steady-state branches were determined at first. For this purpose a parametric continuation of the following boundary problem was performed

$$
\begin{gathered}
\frac{d^{2} \beta}{d \xi^{2}}+\frac{n}{\xi} \frac{d \beta}{d \xi}-\Phi^{2} \frac{\widetilde{r}_{\mathrm{A}}(\beta)}{\widetilde{r}_{\mathrm{Aref}}}=0 \\
\frac{d \beta(0)}{d \xi}=0 \\
\frac{d \beta(1)}{d \xi}=\mathrm{Bi}_{m}[g-\beta(1)]
\end{gathered}
$$

The continuation of the boundary differential problem (21), (22) was done by means of a local parameterization method (Seydel, 2010). Then, a single representative point located on the solution branch and corresponding to $\mathrm{Bi}_{m}=5$ and $\Phi=2$ was selected for the simulation of the pellet dynamics.

\section{RESULTS AND DISCUSSION}

Let $C_{\mathrm{A} 0}^{*}$ denote a bulk reactant concentration corresponding to the steady state of a pellet (Fig. $2 \mathrm{c}$ ). The pellet may depart from the steady state as a result of the change of this value to a different value denoted by $C_{\mathrm{A} 0}(t)$. 
Figure 3 shows transient responses of the catalyst pellet under a sinusoidal disturbance $C_{\mathrm{A} 0}(t)$. Such a disturbance can be caused by intentional perturbations in the feed stream or may be a result of some technological factors.

Figure $3 \mathrm{~b}$ and $3 \mathrm{c}$ report trajectories of the dimensionless reactant concentration at the pellet centre $\beta(0)$ and at its surface $\beta(1)$. For comparison, Fig. 3a illustrates also the dynamics of the pellet in which the sorption phenomenon is neglected. A dashed line refers to the forcing function $g(t)$.


Fig. 3. Response of the catalyst pellet to sinusoidal variations of the bulk reactant concentration $\left(\mathrm{Bi}_{m}=5 ; \Phi=2\right.$; $\left.\omega=0.02 \mathrm{rad} \cdot \mathrm{s}^{-1}\right)$; a) process without adsorption; b) linear isotherm, $K_{a}=0.2 ; \mathrm{c}$ ) Freundlich isotherm,

$$
K_{F}=0.0315 ; v=0.5
$$

Three important dynamic features arise from Fig. 3. When the reactant sorption is neglected in the process, high-amplitude oscillations of concentration involve the entire volume of the pellet, i.e. from the surface to its centre (Fig. 3a). Moreover, like the forcing function, they reach periodically in the whole pellet values $\beta(0)=\beta(1)=0$. The third feature is a large amplitude of these variations. On the other hand, in case of simultaneous chemical reaction and adsorption, a damping of the oscillations takes place within the pellet. Thus the remarkable oscillations involve only a certain portion of the pellet. In fact in the pellet centre their amplitude is negligible. It can be observed that for both adopted sorption isotherms dynamical features of the pellet are similar both qualitatively and quantitatively. Therefore it can be concluded that in the analysis of the pellet dynamics the non-linear sorption isotherm characterized by a small curvature may be replaced by the linear isotherm. 

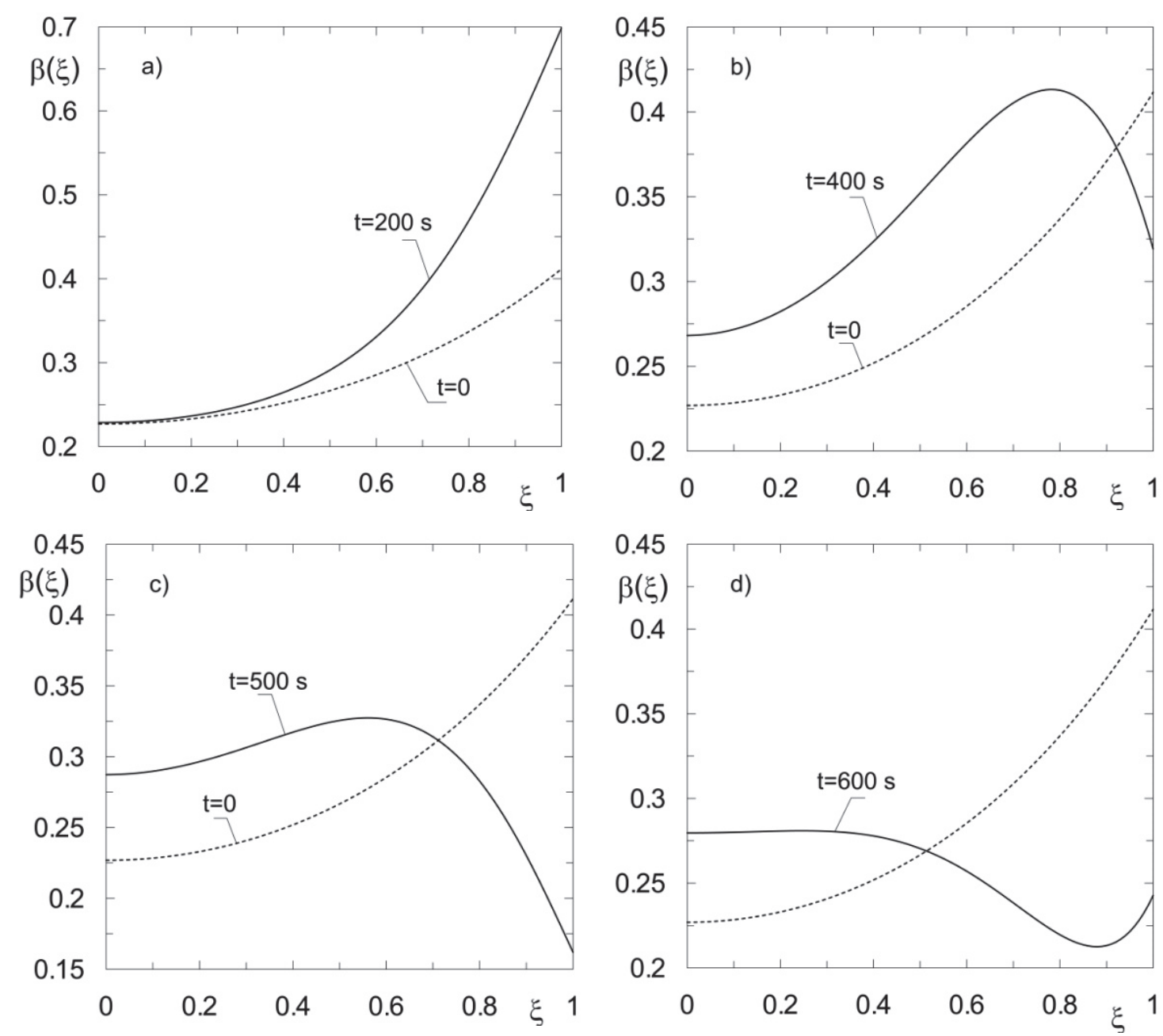

Fig. 4. Transient profiles of dimensionless concentration profiles in the catalyst pellet $\left(\mathrm{Bi}_{m}=5 ; \Phi=2\right.$; $\left.\omega=0.01 \mathrm{rad} \cdot \mathrm{s}^{-1}\right)\left(\right.$ linear isotherm, $\left.K_{a}=0.2\right)$

The sinusoidal variations of the bulk concentration cause reactant concentration extrema within the pellet, i.e. for $0<\xi<1$ (Fig. $4 \mathrm{~b}, \mathrm{c}, \mathrm{d}$ ). Furthermore, as shown in Fig. 4c, with time a periodical inversion of the concentration profile can be observed. The dashed line denotes distribution of the reactant profile corresponding to the steady state.

One of the main objectives of this study was an evaluation of the impact of the bulk reactant perturbation frequency onto the pellet response. It was demonstrated that the amplitude of the oscillations within the pellet, particularly around its centre, depends on the frequency of perturbations. As the frequency $\omega$ increases, the oscillations inside the pellet almost disappear. This feature is demonstrated in Fig. 5.

It can be observed that the oscillation amplitude in the pellet centre becomes negligible when the following inequality holds

$$
\omega>\frac{D_{e}}{L_{p}^{2}}
$$

For the conditions for which the results presented in Fig. 5 were obtained we have $D_{e} / L_{p}^{2}=0.0625$. Then the amplitude at the centre of the pellet, i.e. $\beta(0)$ is lower than $10^{-4}$. 
The kinetic quantity that determines an overall rate of the process in the pellet is its effectiveness factor. It is the quantity of technological importance because it determines the production capacity of the whole catalyst bed. Therefore, apart from the local changes of the concentration within the pellet, transient values of the pellet effectiveness factor was calculated according to the formula

$$
\eta_{0}=\frac{\bar{r}_{\mathrm{A}}}{\widetilde{r}_{\mathrm{Aref}}}
$$

where

$$
\bar{r}_{\mathrm{A}}=\frac{1}{V_{z}} \int_{V_{z}} \widetilde{r}_{\mathrm{A}}(v) d v
$$

Transient values of the effectiveness factor for two selected values of the frequency $\omega$ are presented in Fig. 6. When the reactant sorption is neglected, then the amplitude of the oscillations of $\eta_{0}(t)$ is large and it reaches periodically the value equal to zero. Moreover, it does not depend on the frequency of the external concentration field. It means that the production capacity of the entire bed of the catalyst periodically falls to values close to zero. However it does not approach exactly the value of zero at least due to one reason, namely because of the gas flow through the bed. The second reason can be the mass dispersion in the bed of the catalyst.
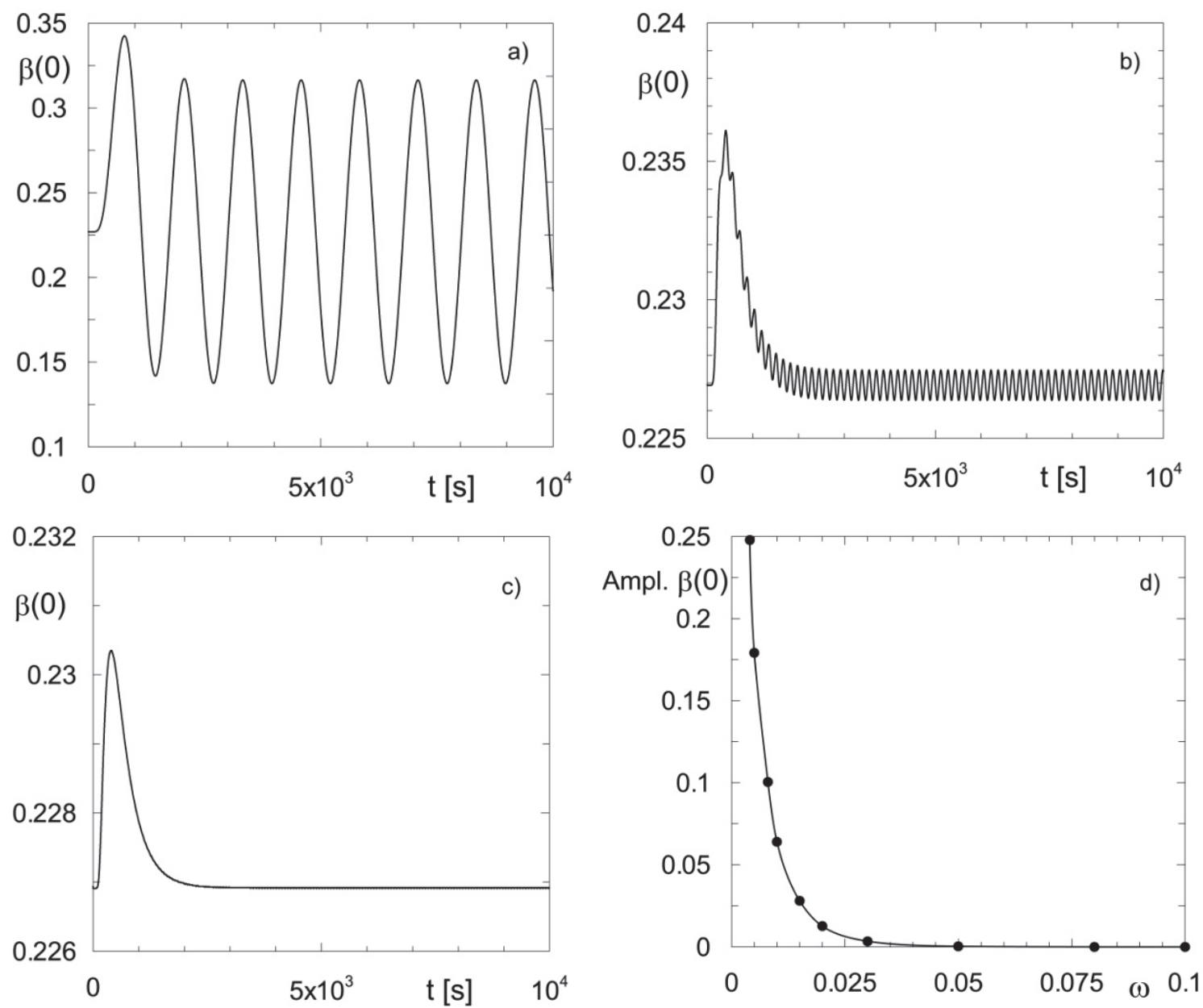

Fig. 5. Amplitude of the oscillations of dimensionless concentration at the pellet center for various frequencies $\omega$, a) $\omega=0.005 \mathrm{rad} \cdot \mathrm{s}^{-1}$; b) $\omega=0.04 \mathrm{rad} \cdot \mathrm{s}^{-1}$; $) \omega=0.1 \mathrm{rad} \cdot \mathrm{s}^{-1}\left(\mathrm{Bi}_{m}=5 ; \Phi=2\right.$; linear isotherm, $\left.K_{a}=0.2\right)$ 

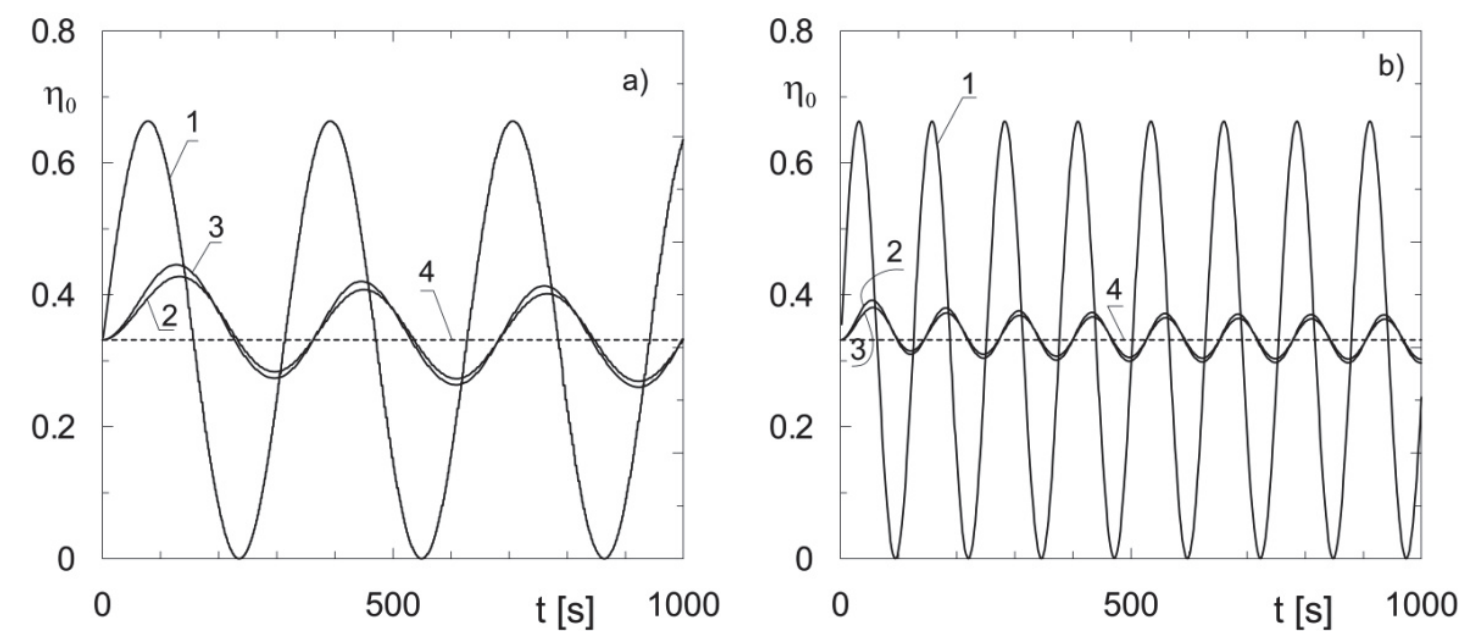

Fig. 6. Transient and average values of an overall effectiveness factor of the pellet $(\Phi=2)$ a) $\omega=0.02 \mathrm{rad} \cdot \mathrm{s}^{-1}$;

b) $\omega=0.05 \mathrm{rad} \cdot \mathrm{s}^{-1} ; 1$ - process without adsorption; 2- linear isotherm, $K_{a}=0.2 ; 3$ - Freundlich isotherm, $K_{F}=0.0315 ; v=0.5 ; 4$ - average values of an effectiveness factor of the pellet

When the reactant sorption occurs then a considerable damping of the oscillations of $\eta_{0}(t)$ takes place. It is the greater the higher the frequency of the bulk reactant concentration variations is. Moreover, compared to the process without sorption, a delay can be observed in the $\eta_{0}(t)$ oscillations. This means that the sorption phenomenon prevents sudden changes of the reactor productivity. In case of the nonlinear sorption isotherm characterized by low curvatures the effectiveness factor values are similar, both qualitatively and quantitatively, to those obtained for the linear isotherm.

Apart from the transient values of the effectiveness factor, its average value was determined from the following formula

$$
\overline{\eta_{0}}=\frac{1}{T} \int_{0}^{T} \eta_{0}(t) d t
$$

The average values of the effectiveness factor of the pellet are plotted in Fig. 6 using a dashed line. It appears that the average values of the effectiveness factor do not depend on the perturbation frequency nor the type of the sorption isotherm.

The results presented above refer to the first order chemical reaction. The same calculations were performed for the chemical reaction characterized by a non-linear kinetics taking $m=2$. Qualitatively similar results which allow to draw analogical conclusions as for the first order reaction were obtained. The differences were only quantitative. Therefore they were not reported in this work.

\section{CONCLUSION}

If a chemical process takes place in porous catalyst pellets then, at transient conditions, a sorption of reactants on the inert support of the pellets may occur. A hypothesis concerning the impact of the sorption of the dynamics of the porous catalyst pellet was demonstrated. For this purpose simulations of the dynamics of a single pellet in which a simultaneous chemical reaction, diffusion and adsorption take place were performed for a certain representative set of technological data. A uniformity of the temperature within the pellet was assumed. Initially a steady state of the pellet was determined and then the pellet was subjected to sinusoidal variations of the bulk reactant concentrations. 
It was demonstrated that the dynamical response of the pellet in which simultaneous adsorption and description of the reactant take place is different than that of the process with negligible sorption phenomena. The pellet response to periodical perturbation for the process without the sorption is characterized by large amplitudes, which qualitatively mimic the forcing function. This affects the dynamics of the entire catalytic reactor because it leads to a significant variation in its productivity. When the process is accompanied by the reactant sorption then significant dumping of oscillations can be seen, involving both local concentrations within the pellet and values of the effectiveness factor $\eta_{0}$.

At low values of the forcing function frequency, remarkable oscillations of the concentration involves an entire volume of the pellet. With increasing frequency the oscillations characterized by high amplitude restrict to the outer part of the pellet and they almost vanish in the centre. A criterion concerning damping of the oscillations in the pellet centre was formulated.

It was demonstrated that amplitudes of a transient effectiveness factor $\eta_{0}(t)$ decrease as the frequency of the forcing function increases. From the point of view of dynamic properties of the entire reactor, this is a preferred phenomenon because in case of significant variations of the bulk reactant concentration it results in a suppression of the fluctuations in the production capacity of the reactor.

A primary practical conclusion emerging from the results presented in this work concerns the approach to the modelling of the catalyst pellet and catalytic reactors. Namely, the models and dynamical simulations of such objects must account for sorption phenomena.

\section{SYMBOLS}

$\begin{array}{ll}B i_{m} & \text { Biot number for mass transfer } \\ C & \text { vector of concentrations } \\ C_{\mathrm{A}} & \text { concentration of reactant A within a catalyst pellet, } \mathrm{kmol} \cdot \mathrm{m}^{-3} \\ C_{\mathrm{A} r e f} & \text { reference concentration of reactant A, } \mathrm{kmol} \cdot \mathrm{m}^{-3} \\ C_{\mathrm{A} 0} & \text { bulk concentration of reactant A, } \mathrm{kmol} \cdot \mathrm{m}^{-3} \\ C_{\mathrm{A} 0}^{*} & \text { bulk concentration of reactant A at steady state, } \mathrm{kmol} \cdot \mathrm{m}^{-3} \\ D_{e} & \text { effective diffusion coefficient, } \mathrm{m}^{2} \cdot \mathrm{s}^{-1} \\ d_{p} & \text { diameter a catalyst pellet, } \mathrm{m} \\ g & \text { dimensionless forcing function } \\ k & \text { rate constant of a chemical reaction, } \mathrm{m}^{3(m-1)} \cdot \mathrm{s}^{-1} \cdot \mathrm{kmol}^{(1-m)} \\ k_{s} & \text { mass transfer coefficient between gas phase and catalyst pellet, } \mathrm{m}^{-1} \cdot \mathrm{s}^{-1} \\ K_{a} & \text { slope of a sorption equilibrium curve, } \mathrm{m}^{3} \cdot \mathrm{kg}^{-1} \\ K_{F} & \text { Freundlich constant } \\ L_{p} & \text { characteristic length of a catalyst pellet, } \mathrm{m} \\ m & \text { order of chemical reaction } \\ n & \text { shape factor of a catalyst pellet } \\ N & \text { number of grid nodes } \\ q & \text { amount of reactant adsorbed on inner inert surface of a catalyst pellet, } \mathrm{kmol} \cdot \mathrm{kg}^{-1} \\ r_{A} & \text { rate of a chemical reaction, kmol } \cdot \mathrm{m}^{-3} \cdot \mathrm{s}^{-1} \\ \widetilde{r}_{\mathrm{A}} & \text { modified rate of a chemical reaction, i.e. } \widetilde{r}_{\mathrm{A}}(\beta)=r_{\mathrm{A}}\left(C_{\mathrm{A}}\right) / C_{\mathrm{Aref}}, \mathrm{s}^{-1} \\ \widetilde{r}_{\mathrm{Aref}} & \text { modified rate of a chemical reaction evaluated at } C_{\mathrm{Aref}}, \text { i.e. } \widetilde{r}_{\mathrm{Aref}}=C_{\mathrm{Aref}}^{m-1} \cdot k, \mathrm{~s}^{-1} \\ t & \text { time, } \mathrm{s} \\ T & \text { period of oscillations, s } \\ x & \text { current coordinate in a catalyst pellet, } \mathrm{m}\end{array}$




\section{Greek symbols}

$\beta \quad$ dimensionless concentration in a catalyst pellet

$\Gamma_{m}$

mass capacitance term, $\mathrm{s}^{-1}$

$\varepsilon_{p}$

porosity of a catalyst pellet

$\eta_{0}$

overall effectiveness factor of a catalyst pellet

$v$

$\xi$

$\rho_{p}$

$\rho_{0}$

$\Phi$ exponent in the Freundlich equation dimensionless coordinate in a pellet density of a catalyst pellet, $\mathrm{kg} \cdot \mathrm{m}^{-3}$

$\omega$ density of solid material, $\mathrm{kg} \cdot \mathrm{m}^{-3}$ Thiele modulus

\section{Subscripts}

$\begin{array}{ll}0 & \text { refers to conditions in a bulk gas } \\ A & \text { refers to reactant } A \\ m & \text { refers to mass } \\ p & \text { refers to catalyst pellet } \\ s & \text { refers to catalyst pellet surface }\end{array}$

\section{REFERENCES}

Burghardt A., Berezowski M., 2003. Periodic solutions in a porous catalyst pellet - Homoclinic orbits. Chem. Eng. Sci., 58, 2657-2670. DOI: 10.1016/S0009-2509(03)00120-9.

Dietrich W., Lawrence P.S., Grünewald M., Agar D.W., 2005. Theoretical studies on multifunctional catalysts with integrated adsorption sites. Chem. Eng. J., 107, 103-111. DOI: 10.1016/j.cej.2004.12.016.

Gear C. W., 1971. Algorithm 407: DIFSUB for solution of ordinary differential equations [D2]. Mag. Commun. $A C M, 14,185-190$. DOI: $10.1145 / 362566.362573$.

Grünewald M., Agar D.W., 2004. Enhanced catalyst performance using integrated structured functionalities. Chem. Eng. Sci., 59, 5519-5526. DOI: 10.1016/j.ces.2004.07.072.

Il' in A., Luss D., 1992. Wrong-way behavior of packed bed reactors: Influence of reactant adsorption on support. AIChE J., 38, 1609-1617. DOI: 10.1002/aic.690381011.

Lee H.H., 1985. Heterogeneous reactor design. Butterworth Publishers, Boston - Toronto.

Lugo E.L., Wilhite B.A., 2016. A theoretical comparison of multifunctional catalyst for sorption-enhanced reforming process. Chem. Eng. Sci., 150, 1-15. DOI: 10.1016/j.ces.2016.04.011.

Luss D., 1974. The influence of capacitance terms on the stability of lumped and distributed parameter systems. Chem. Eng. Sci., 29, 1832-1836. DOI: 10.1016/0009-2509(74)87045-4.

Ray W.H., Hastings S.P., 1980. The influence of the Lewis number on the dynamics of chemically reacting systems. Chem. Eng. Sci., 35, 589-595. DOI: 10.1016/0009-2509(80)80007-8.

Seydel R., 2010. Practical bifurcation and stability analysis. Springer, New York London.

Shampine L. F., 1994. ODE solvers and the method of lines. Numer. Methods Partial Differential Eq. 10, 739-755. DOI: $10.1002 /$ num. 1690100608.

Szarawara J., Skrzypek J., 1980. Podstawy inżynierii reaktorów chemicznych. WNT, Warszawa.

Szukiewicz M. K., 2002. An approximate model for diffusion and reaction in a porous pellet. Chem. Eng. Sci., 57, 1451-1457. DOI: 10.1016/S0009-2509(02)00055-6. 